\title{
Asymmetric STN DBS for FOG in Parkinson's disease: A pilot trial
}

Sara Meoni ${ }^{\mathrm{a}, \mathrm{b}}$, Bettina Debû ${ }^{\mathrm{b}}$, Pierre Pelissier ${ }^{\mathrm{a}}$, Emma Scelzo ${ }^{\mathrm{a}}$, Anna Castrioto ${ }^{\mathrm{a}, \mathrm{b}}$, Eric

Seigneuret $^{b, c}$, Stephan Chabardes ${ }^{b, c}$, Valerie Fraix ${ }^{a, b}$, Elena Moro ${ }^{a, b}$

${ }^{a}$ Division of Neurology, Movement Disorders Unit, CHU of Grenoble, Grenoble Alpes University, Avenue Maquis du Grésivaudan, 38700 Grenoble, France

${ }^{\mathrm{b}}$ Grenoble Alpes University, INSERM U1216, Grenoble Institut Neuroscience, 38000 Grenoble, France

${ }^{\mathrm{c} D}$ Department of Neurosurgery, CHU of Grenoble, Grenoble Alpes University, Avenue Maquis du Grésivaudan, 38700 Grenoble, France

Corresponding author:

Elena Moro, $\mathrm{MD}, \mathrm{PhD}$

Movement Disorders Unit

Department of Psychiatry and Neurology

Centre Hospitalier Universitaire de Grenoble

BP217 38043 Grenoble CEDEX 09 France

E-mail: elenamfmoro@gmail.com 
Title: 64 characters

Abstract: 250 words

Paper: 2739 words

References: 29 words

Tables/ Figures: 3 tables, 1 figure

Supplementary material: 1 table, 2 figures.

Study Funding: This project was funded in 2013 by a grant from Délégation à la Recherche Clinique et à l'Innovation (DRCI), Centre Hospitalier Universitaire de Grenoble, France.

\section{Disclosure Statement}

Sara Meoni reports no disclosures.

Bettina Debû reports no disclosures.

Pierre Pelissier reports no disclosures.

Emma Scelzo reports no disclosures.

Anna Castrioto has received a grant from France Parkinson Foundations, reimbursement of travel expenses to scientific meetings from ELIVIE, Medtronic and Boston Scientific.

Eric Seigneuret reports no disclosures.

Stephan Chabardes received honoraria as consultant from Medtronic, Zimmer Biomet and Boston Scientific.

Valerie Fraix received honoraria as consultant from AbbVie France.

Elena Moro has received honoraria for lecturing from Medtronic and Bial. She has received honoraria as consultant from Medtronic and Newronika. She has received research grant from Merz. 


\begin{abstract}
Background: In Parkinson's disease (PD), freezing of gait (FOG) is a highly disabling gait disorder. Though deep brain stimulation (DBS) of the subthalamic nucleus (STN) is an efficient treatment for advanced PD, the management of STN DBS refractory FOG remains challenging.
\end{abstract}

Objective: To evaluate the long-term impact on FOG of unilateral stimulation reduction in PD treated with bilateral STN DBS.

Methods: Patients with bilateral STN DBS for at least one year and refractory FOG were included in a randomized, double blind, cross-over clinical trial. They were randomized to chronic (CHR) vs. experimental (EXP) stimulation (30\% amplitude reduction contralateral to the least affected body side), each condition for 4 weeks. Gait and FOG were assessed both in the OFF and ON medication conditions. Primary outcome was the difference in the FOG percentage during gait assessment and in a composite gait score in CHR vs. EXP stimulation.

Results: The study was stopped early for futility. Of the 12 patients included, eight dropped out because of re-emerging of PD symptoms. In the four patients who sustained the experimental condition, the FOG percentage did not improve, whether in the OFF (CHR: 13.4\% vs. EXP: 16.8\%) or in the ON (CHR: 19.5\% vs. EXP: 19.8\%) medication condition. There was no change in the composite gait score (CHR: $5.5 \pm 1.3$ vs. EXP: $6.3 \pm 3.3$ ).

Conclusions: Most patients did not tolerate the unilateral amplitude reduction of STN DBS in the long-term. Moreover, this strategy failed to improve FOG in patients who could sustain the procedure.

ClinicalTrial.gov identifier: NCT02704195

Keywords: subthalamic, DBS, freezing of gait, Parkinson's disease, asymmetric stimulation. 


\section{Introduction}

In Parkinson's disease (PD), freezing of gait (FOG) is a common and disabling gait disorder, described as an episodic, short-lasting difficulty to perform effective stepping [1]. It may occur most frequently at step initiation and during turning. Some factors such as narrow spaces, mental distraction, stress, and double tasks may induce or worsen FOG [2]. It has been reported that up to 90\% of advanced PD patients are affected by this gait disorder [3]. FOG is associated with disease severity and poor quality of life [4], and it is an independent risk factor for falls [5].

The pathophysiology of FOG is still largely unknown. Abnormalities in gait coordination, symmetry and rhythmicity, such as increased variability of gait cycle [6], temporal step dysregulation [7], and alterations of step symmetry [8] have been described in patients with FOG compared to non-freezers. These abnormalities could lead to a deep disorganization of gait, causing FOG, although the exact role of these gait parameters in the genesis of FOG is not clear.

FOG is difficult to treat, since it can be levodopa-resistant and, in some cases, induced by levodopa [9]. Though high frequency deep brain stimulation (DBS) of the subthalamic nucleus (STN) is an efficient treatment for advanced PD [10], its effects on FOG remain controversial. L-dopa responsive FOG may be improved by STN DBS [11], but it has also been reported that STN DBS can induce or worsen FOG in some patients [12]. Moreover, with the disease progression, axial signs including gait issues become resistant to treatment, either dopaminergic or STN DBS therapy [13]. Several neuromodulation strategies have been attempted to alleviate FOG, including low frequency stimulation and stimulation of targets other than STN (i.e., the pedunculopontine nucleus, the substantia nigra reticulata, and the spinal cord) [14].

Based on the hypothesis that FOG is related to an abnormal inter limb coordination $[7,8]$, unilateral reduction of stimulation amplitude to correct step asymmetry has been investigated in one small open, acute study [15]. The authors concluded that reducing stimulation amplitude for the STN 
contralateral to the leg with the longer step could normalise limb coordination, leading to FOG improvement. Yet, this study raises several issues, such as the very short duration of the parameters' change (30 minutes) that does not allow to conclude on the therapeutic utility of this strategy in daily life [16]. Moreover, a long-term reduction in the stimulation intensity could lead to unbearable worsening of other motor symptoms, like tremor. To address these gaps, we conducted a randomized, double blind, cross-over clinical trial, aimed at assessing the long-term efficacy of unilateral STN stimulation reduction on FOG in PD patients treated with bilateral STN DBS.

\section{METHODS}

\subsection{Patients}

A monocentric, randomized, double-blind, cross-over, controlled clinical trial was conducted at the Movement Disorders Unit, Grenoble Alpes University Hospital (France), between April 2014 and March 2016. Main inclusion criteria were as follows: patients with advanced PD (UK Parkinson's disease Society Brain Bank criteria) who had bilateral STN surgery for at least one year; age between 30 and 75 years; mild to severe FOG, as scored by the MDS-UPDRS III 3.11 item subscore [17], in the ON medication condition despite optimisation of medication and parameters of stimulation. Patients with active psychosis, severe depression, cognitive impairment (Mattis Dementia Rating Scale [18] score $<130$ ) and any other medical or neurologic condition interfering with gait were excluded.

The details of STN DBS surgery are reported elsewhere [19].

\subsection{Standard protocol approvals, registrations, and patient consents}

The study was sponsored by Centre Hospitalier Universitaire de Grenoble, approved by the French regulatory authorities and national ethical committee, and conducted in accordance with 
the Declaration of Helsinki [20] and Good Clinical Practice Guidelines. Written informed consent was obtained from all study participants (ClinicalTrials.gov NCT NCT02704195).

\subsection{Randomization and blinding}

Eligible patients were randomized to receive chronic parameters stimulation (CHR) or experimental parameters stimulation (EXP) [21]. The EXP condition consisted in a 30\% reduction of the stimulation amplitude at the STN contralateral to the least affected body side at time of surgery. The sequence of the conditions was generated by a computerized application (http://randomnumbergenerator.intemodino.com/fr/). All patients, the neurologists (SM, ES) and gait researcher (BD) who performed the motor assessment, gait recording and off-line analysis were blinded to the stimulation condition.

\subsection{Procedure and clinical evaluations}

The study was designed as a 12-week trial, with a 4-week initial open phase followed by an 8 -week blinded phase, with a total of six scheduled visits (screening, baseline, week 2, 4, 8, and 12), as illustrated in Supplementary Material (figure 1S). During the open phase, the tolerability of the EXP setting was tested for each body side separately, to ensure that the patients remained truly blind to the parameters setting in the subsequent randomized double blinded phase, and thus reduce possible placebo effects. No changes in dopaminergic medications and physiotherapy (if present) were allowed during the study.

At baseline, all patients underwent motor and gait assessments under chronic stimulation parameters, after a 12-hour withdrawal of dopaminergic treatment (MED OFF/STIM ON condition) and after a supraliminal (50\%) levodopa dose intake (MED ON/STIM ON condition) [19]. Subsequently, the EXP setting was applied to the right STN (R-stim). A remote control was given to patients to increase the stimulation back to the chronic parameters at home in case of intolerance to the 
stimulation reduction.

At week 2, a motor assessment was performed under chronic dopaminergic treatment and R-stim condition. Then the EXP setting was applied to the left STN (L-stim), whereas the chronic amplitude was restored in the right STN.

At week 4, a motor assessment was performed under chronic medication and L-stim condition. Then, if the patient had tolerated the stimulation reduction contralateral to the least affected side, the unblinded neurologist (EM) set the parameters according to the randomisation sequence: CHR or EXP.

At week 8, blinded motor and gait assessments under MED OFF/STIM ON and MED ON/ STIM ON (CHR or EXP condition) were performed before switching the stimulation condition (EM). The same blinded assessment was performed again at week 12. At this point, patients were asked which parameter settings (EXP - CHR) had best alleviated their FOG and were offered to keep these parameters as chronic settings. All motor and gait blinded assessments were videotaped.

Motor assessments were performed by neurologists (SM, ES) using the Movement Disorders Society Unified Parkinson Disease Rating Scale (MDS-UPDRS) part III [17].

Gait assessment. Patients performed a walking test including walking on an 8-m walkway at their normal pace, and under FOG provoking circumstances such as narrow spaces, half- and full turns to the right and to the left, as well as a dual cognitive-motor task. Five trials were randomly administered. Inner soles (Stride Analyzer, B\&L Engineering, Santa Ana, CA, USA) containing four footswitches (one each for the heel, big toe, first and fifth metatarsal heads), were placed in the patients' shoes. The foot-floor contact data were collected using a telemetric acquisition system (Noraxon Telemyo 2400, Scottsdale, USA) with video recording synchronization. The beginning and end of each freezing episode were marked on the foot contact data before the files were exported and 
further processed to quantify the duration of freezing during the walking test.

The MDS-UPDRS part II [17], the FOG questionnaire (FOG-Q) [22] and the PDQ39 questionnaire (PDQ39-Q) [23] were completed at each study visit. Stimulation parameters were verified at each visit. Safety assessment included adverse events and reasons for discontinuation.

\subsection{Statistical analysis}

The primary outcome was the difference between the CHR and EXP conditions in the percent of FOG during the gait test duration (summed duration of FOG over total duration of gait assessment $\mathrm{X} 100$ ), and in the composite gait score computed as the sum of the MDS-UPDRS part II items 12 (gait and balance) and 13 (FOG), and the MDS-UPDRS part III item 10 (gait) and 11 (FOG) subscores. Secondary outcomes were the difference between the CHR and EXP condition in the MDS-UPDRS part III and part II total scores, as well as in the lateralized upper limb MDS-UPDRS III subscores for rigidity (item 3.3), akinesia (items 3.4, 3.5, 3.6), and tremor (items 3.15, 3.16, 3.17, 3.18). We also compared to the FOG-Q and PDQ-SI (PDQ39 Summary Index Score) between the two conditions. According to initial statistical power calculation, 24 patients were expected to provide $90 \%$ power to detect a mean difference of $40 \%$ in FOG (with $30 \%$ inter-subject variability) at $\mathrm{p} \leq 0.05$. For all analyses, the non-parametric Wilcoxon test for paired samples was chosen to compare the two stimulation settings under each medication condition. The statistical threshold was set at $\mathrm{p} \leq 0.05$. Descriptive statistics (mean and standard deviation) were computed for all outcome variables.

Data availability

Anonymized data will be shared on request from any qualified investigator.

\section{RESULTS}


Twelve patients were enrolled in the study. Of the 12 patients included, six presented with akinetorigid PD type, three had a tremor-dominant phenotype, and three presented with a mixed PD phenotype. Eight patients could not tolerate the chronic unilateral reduction of stimulation voltage in the STN contralateral to the least affected body side during the open phase. Indeed, after a few days at most (mean $3.5 \pm 1.2$ days, range 1-5 days), they reported an unbearable worsening of one or more parkinsonian symptoms: hemi-dystonia (2), tremor (4), FOG (4), hypophonia, and/or fatigue (2). Thus, only four patients (\# 2, 5, 11 and 12) could enter and complete the blinded phase of the study. Of these four, three patients had a mixed PD phenotype and one a tremor-dominant phenotype. The trial was stopped earlier for futility by the principal investigator. The flow diagram is illustrated in Supplemental Material (figure 2S).

The demographical data, composite gait score and questionnaire data at baseline of the whole study population are presented on table 1. The patients who tolerated the EXP condition tended to be older and have less-levodopa responsive motor symptoms than the patients who did not sustain the condition. Table 2 shows the DBS settings for the CHR and EXP conditions for the study population. All the patients were on constant Voltage mode stimulation.

\subsection{Outcomes}

Primary outcomes. For the four patients who completed the study, the mean percentage of FOG was $13.4( \pm 15.6)$ under the CHR condition vs. $16.8( \pm 18.9)$ under the EXP condition in the OFF medication condition (figure 1A). In the ON medication condition, the mean percentage of FOG was 19.5 ( \pm 26.2) under the CHR condition vs. $19.8 \pm 23.6$ under the EXP condition (figure 1B). The composite gait score was $5.5 \pm 1.3$ under the CHR condition and $6.3 \pm 3.3$ under the EXP condition (figure 1C). 
Secondary outcomes. There was no difference under CHR vs. EXP setting in the MDS-UPDRS part III total score in both the OFF and ON medication condition, MDS-UPDRS II total score, FOG-Q score and PDQ-SI score, as shown in table 3.

\section{DISCUSSION}

This double-blinded study assessed the long-term tolerability and efficacy of asymmetric amplitude reduction of STN DBS to improve FOG in PD patients. Of 12 patients included, only four could complete the study. Indeed, unbearable side effects related to the reduced amplitude started on average three days after the reduction of the stimulation amplitude contralateral to the least affected side. Therefore, the principal investigator (EM) decided to interrupt the study before reaching the planned number of inclusions. In the four patients who completed the study, unilateral STN DBS amplitude reduction failed to improve FOG.

Our results failed to demonstrate any improvement in FOG during objective testing under the EXP conditions, whatever the medication condition. Furthermore, the composite gait score and the FOGQ score did not improve either under the EXP condition, confirming the results of objective gait assessment.

Our findings are in contrast with a previous study investigating the effect of unilateral stimulation amplitude reduction on FOG in PD patients with STN DBS [15]. In this open study, the STN stimulation amplitude was reduced by $50 \%$ in the STN contralateral to the side with longer step length before gait and FOG assessment. The working hypothesis was that FOG would improve through gait symmetry restoration. In the 13 PD patients included, frequency and duration of FOG improved after 15 minutes under low amplitude stimulation at one STN side. However, this study did not assess the long-term clinical feasibility of such a change in parameter setting. As already demonstrated, delayed effects on clinical signs after changing STN DBS parameters can take several hours/days to appear $[16,24]$. In our patients, subjective worsening of parkinsonian signs appeared 
between 1 and 5 days after reduction of unilateral stimulation amplitude. For eight patients, this clinical worsening was so severe that they dropped out of the study. Therefore, a reliable clinical evaluation of changes in DBS parameters implies that these changes be applied for a prolonged enough time before assessment. Our results suggest that it should be at least 24 hours. Moreover, a large reduction in the stimulation amplitude is unlikely to be tolerated in patients with optimized chronic medical and stimulation therapy [25]. For this reason, we adopted a conservative $30 \%$ reduction of the amplitude instead of 50\%. Yet, even this smaller reduction was not tolerated by most the patients. Finally, because of the well-known sensitivity of FOG to psychological and cognitive factors [2], results can greatly differ between open and blind assessments.

Therefore, it remains to be demonstrated that asymmetric modulation of STN stimulation has an impact on gait coordination and FOG in PD [7], although a role of an asymmetric basal ganglia output in the genesis of axial symptoms and gait issues could be supported by a dominant-STN [26]. Indeed, two recent studies $[27,28]$ have investigated the dominance phenomenon of the STN on gait function in PD patients with bilateral DBS. Overall, the results support the hypothesis of a dominant STN on gait, in particular, right-sided stimulation might be superior in improving axial motor function [27].

No increase in dopaminergic medication was allowed during the study, as in some patients gait and FOG were partially levodopa-responsive. Indeed, changes on medication, in addition to possible side effects such as dyskinesia or poor tolerance, would have modified FOG, and confounded the effects of stimulation on gait and FOG.

The blinding was not compromised. As shown in supplementary material (Table-S), in those patients who completed the trial, the difference in the appendicular motor score in EXP vs. CHR condition was mild so to let them tolerate the voltage reduction and enter the blinded phase. As such, patients and investigators did not specifically notice the mild worsening, as it was not greater than the common motor fluctuations observed on a day to day basis in PD patients. 
Our study has some limitations. The main limitation concerns the small sample of patients who completed the study, although related to the high rate of drop-outs. Psychological factors could have contributed to the negative outcome of the study. Indeed, the motivation of patients to perform the gait test to the best could have improved their performance, underrating the severity of FOG during the study. Yet, this would have affected both stimulation conditions as patients were blinded to the parameters settings. Another possible bias is related to the experimental protocol. STN stimulation amplitude was reduced contralateral to the least affected side, assuming that this would correspond to the leg with the longer step. Our equipment did not unable reliable assessment of step length. It cannot be excluded that step length in the least affected side was shorter than in the contralateral side in our patients. However, amplitude reduction was tested on both sides during the open phase of the study and no patients reported a subjective improvement of FOG when the amplitude was reduced contralateral to the most affected side. A further limitation of our study is the use of a non-validated composite gait score based on MDS-UPDRS items as an outcome measure. However, numerous PDrelated studies utilize MDS-UPDRS-based composite scores as their outcome measure [29].

\section{CONCLUSIONS}

To the best of our knowledge, this is the first double blinded clinical trial to investigate the long-term efficacy of asymmetric STN DBS reduction for FOG in PD patients under chronic conditions. This strategy failed to improve FOG in those patients who could sustain the amplitude reduction. Moreover, this DBS strategy was not tolerated by most patients because of re-emerging of other symptoms.

The management of STN DBS refractory FOG remains challenging, and new long-term stimulation strategies need to be found or designed.

\section{Acknowledgements}


The authors thank Dr. A. Al-Dakheel for her assistance in patients' evaluation and all patients for partecipating to the study.

\section{References}

[1] Nutt JG, Bloem BR, Giladi N, et al. Freezing of gait: moving forward on a mysterious clinical phenomenon. Lancet Neurol 2011; 10:734-44.

[2] Giladi N, Nieuwboer A. Understanding and treating freezing of gait in parkinsonism, proposed working definition, and setting the stage. Mov Disord 2008; 23:S423-S425.

[3] Cilia R, Cereda E, Klersy C, Canesi M, Zecchinelli AL, Mariani CB, et al. Parkinson's disease beyond 20 years. J Neurol Neurosurg Psychiatry 2015; 86:849-55.

[4] Walton CC, Shine JM, Hall JM, O'Callaghan C, Mowszowski L, Gilat M, et al. The major impact of freezing of gait on quality of life in Parkinson's disease. J Neurol 2015; 262:108-15.

[5] Perez-Lloret S, Negre-Pages L, Damier P, et al. Prevalence, determinants, and effect on quality of life of freezing of gait in Parkinson disease. JAMA Neurol 2014; 71: 884-890.

[6] Nieuwboer A, Dom R, De Weerdt W, Desloovere K, Janssens L, Stijn V. Electromyographic profiles of gait prior to onset of freezing episodes in patients with Parkinson's disease. Brain 2004; 127: $1650-1660$.

[7] Plotnik M, Giladi N, Hausdorff JM. Bilateral coordination of walking and freezing of gait in Parkinson's disease. Eur J Neurosci 2008; 27:1999-2006.

[8] Plotnik M, Giladi N, Balash Y, Peretz C, Hausdorff JM. Is freezing of gait in Parkinson's disease related to asymmetric motor function? Ann Neurol 2005; 57:656-63.

[9] Espay AJ, Fasano A, van Nuenen BF, et al. “On” state freezing of gait in Parkinson's disease: A paradoxical levodopa-induced complication. Neurology 2012; 75:454-7.

[10] Deuschl G, Agid Y. Subthalamic neurostimulation for Parkinson's disease with early fluctuations: balancing the risks and benefits. Lancet Neurol 2013; 12: 1025-1034. 
[11] Vercruysse S, Vandenberghe W, Munks L, Nuttin B, Devos H, Nieuwboer A. Effects of deep brain stimulation of the subthalamic nucleus on freezing of gait in Parkinson's disease: a prospective controlled study. J Neurol Neurosurg Psychiatry 2014; 85: 871-877.

[12] Fleury V, Pollak P, Gere J, Tommasi G, Romito L, Combescure C, et al. Subthalamic stimulation may inhibit the beneficial effects of levodopa on akinesia and gait. Mov Disord 2016; 31:1389-97.

[13] Debû B, De Oliveira Godeiro C, Lino JC, Moro E. Managing Gait, Balance, and Posture in Parkinson's Disease. Curr Neurol Neurosci Rep. 2018; 18:23.

[14] Schlenstedt C, Shalash A, Muthuraman M, Falk D, Witt K, Deuschl G. Effect of high-frequency subthalamic neurostimulation on gait and freezing of gait in Parkinson's disease: a systematic review and meta-analysis. Eur J Neurol 2017; 24:18-26.

[15] Fasano A, Herzog J, Seifert E, Stolze H, Falk D, Reese R, et al. Modulation of gait coordination by subthalamic stimulation improves freezing of gait. Mov Disord 2011 Apr; 26:844-51.

[16] Temperli P, Ghika J, Villemure JG, Burkhard PR, Bogousslavsky J, Vingerhoets FJ. How do parkinsonian signs return after discontinuation of subthalamic DBS? Neurology 2003; 60:78-81. [17] Goetz CG, Tilley BC, Shaftman SR, Stebbins GT, Fahn S, Martinez-Martin P, et al. . Movement Disorder Society-sponsored revision of the Unified Parkinson's Disease Rating Scale (MDSUPDRS): scale presentation and clinimetric testing results. Mov Disord 2008; 23:2129-70. [18] Mattis, S. Mental status examination for organic mental syndrome in the elderly patient. In: Bellak, T, Karasi, TB (Eds.), Geriatric psychiatry. New York: Crane and Stratten; 1976: 77-121. [19] Krack P, Batir A, Van Blercom N, et al. Five-year follow-up of bilateral stimulation of the subthalamic nucleus in advanced Parkinson's disease. N Engl J Med 2003; 349:1925-1934. [20] World Medical Association. World Medical Association Declaration of Helsinki: ethical principles for medical research involving human subjects. JAMA 2013; 310:2191-2194. 
[21] Schulz KF, Grimes DA. Generation of allocation sequences in randomised trials: chance, not choice. Lancet 2002; 9305: 515-19.

[22] Giladi N, Shabtai H, Simon ES, Biran S, Tal J, Korczyn AD. Construction of freezing of gait questionnaire for patients with Parkinsonism. Parkinsonism Relat Disord 2000; 6:165-170. [23] Auquier P, Sapin, C, Ziegler M, Tison, F, Destee A, Dubois B. Validation of the French language version of the Parkinson's Disease Questionnaire - PDQ-39. Rev Neurol (Paris) 2002; 158: 41-50.

[24] Ricchi V, Zibetti M, Angrisano S, Merola A, Arduino N, Artusi CA,et al.Transient effects of 80 Hz stimulation on gait in STN DBS treated PD patients: a 15 months follow-up study. Brain Stimul $2012 ; 5: 388-92$.

[25] Moro E, Esselink RJ, Xie J, Hommel M, Benabid AL, Pollak P. The impact on Parkinson's disease of electrical parameter settings in STN stimulation. Neurology 2002; 59:706-13. [26] Castrioto A, Lozano AM, Poon YY, Lang AE, Fallis M, Moro E. Ten-year outcome of subthalamic stimulation in Parkinson's disease: a blinded evaluation. Archives of Neurology 2011; 68:1550-1556.

[27] Lizarraga KJ, Jagid JR, Luca CC. Comparative effects of unilateral and bilateral subthalamic nucleus deep brain stimulation on gait kinematics in Parkinson's disease: a randomized, blinded study. J Neurol 2016; 263:1652-6.

[28] Rizzone MG, Ferrarin M, Lanotte MM, Lopiano L, Carpinella I. The Dominant-Subthalamic Nucleus Phenomenon in Bilateral Deep Brain Stimulation for Parkinson's Disease: Evidence from a Gait Analysis Study. Front Neurol 2017; 8:575.

[29] Makkos A, Kovács M, Aschermann Z, Harmat M, Janszky J, Karádi K, et al. Are the MDS-UPDRS-Based Composite Scores Clinically Applicable? Mov Disord. 2018; 33:835-839. 
Table 1. Baseline characteristics of the whole study population.

\begin{tabular}{|c|c|c|c|c|c|c|c|c|c|c|c|c|}
\hline $\begin{array}{l}\mathrm{Pt}, \mathrm{n} . \\
(\mathrm{M} / \mathrm{F})\end{array}$ & $\begin{array}{l}\text { Age } \\
\text { (yrs) }\end{array}$ & $\begin{array}{c}\text { Disease } \\
\text { duration } \\
\text { (yrs) }\end{array}$ & $\begin{array}{c}\text { Most } \\
\text { affected } \\
\text { body } \\
\text { side } \\
\text { (R/L) }\end{array}$ & $\begin{array}{c}\text { DBS } \\
\text { duration } \\
\text { (yrs) }\end{array}$ & $\begin{array}{c}\text { FOG } \\
\text { duration } \\
(\mathrm{yrs})\end{array}$ & $\begin{array}{l}\text { LEDD } \\
(\mathrm{mg})\end{array}$ & $\begin{array}{c}\text { MDS } \\
\text { UPDRS II } \\
\text { score } \\
(\max 52)\end{array}$ & $\begin{array}{c}\text { MDS } \\
\text { UPDRS III } \\
\text { Med OFF } \\
\text { score } \\
(\max 132)\end{array}$ & $\begin{array}{c}\text { MDS } \\
\text { UPDRS III } \\
\text { Med ON } \\
\text { score } \\
(\max 132)\end{array}$ & $\begin{array}{l}\text { FOG-Q } \\
\text { score } \\
(\max 24)\end{array}$ & $\begin{array}{l}\text { Composite } \\
\text { gait score } \\
(\max 12)\end{array}$ & $\begin{array}{c}\text { PDQ-SI } \\
\text { score }\end{array}$ \\
\hline $1(\mathrm{M})$ & 65 & 15 & $\mathrm{~L}$ & 7 & 6 & 600 & 10 & 41.5 & 25.5 & 23 & 4 & 3.8 \\
\hline $3(\mathrm{M})$ & 62 & 14 & $\mathrm{R}$ & 4 & 8 & 880 & 24 & 31 & 25 & 17 & 5 & 8.4 \\
\hline $4(\mathrm{~F})$ & 60 & 11 & $\mathrm{R}$ & 5 & 4 & 220 & 15 & 37 & 27 & 18 & 6 & 9.9 \\
\hline $6(\mathrm{~F})$ & 57 & 7 & $\mathrm{~L}$ & 1 & 3 & 450 & 26 & 41 & 15 & 27 & 7 & 10.9 \\
\hline $7(\mathrm{M})$ & 64 & 14 & $\mathrm{R}$ & 3 & 3 & 630 & 21 & 41 & 23 & 20 & 4 & 3.6 \\
\hline $8(\mathrm{M})$ & 67 & 21 & $\mathrm{~L}$ & 7 & 5 & 525 & 22 & 36 & 18.5 & 23 & 5 & 8.4 \\
\hline $9(\mathrm{M})$ & 62 & 11 & $\mathrm{~L}$ & 1 & 3 & 925 & 34 & 51 & 19 & 28 & 10 & 12.6 \\
\hline $10(\mathrm{~F})$ & 68 & 17 & $\mathrm{R}$ & 8 & 4 & 250 & 26 & 33 & 23 & 22 & 7 & 10 \\
\hline $\begin{array}{l}\text { Mean } \\
(\mathrm{SD})\end{array}$ & $\begin{array}{l}63.1 \\
(3.6)\end{array}$ & $\begin{array}{l}13.8 \\
(4.2)\end{array}$ & $4 \mathrm{R} / 4 \mathrm{~L}$ & $\begin{array}{l}4.5 \\
(2.7)\end{array}$ & $\begin{array}{l}4.5 \\
(1.8)\end{array}$ & $\begin{array}{c}560 \\
(258)\end{array}$ & $\begin{array}{l}22.3 \\
(7.3)\end{array}$ & $\begin{array}{l}38.9 \\
(6.2)\end{array}$ & $\begin{array}{c}22 \\
(4.1)\end{array}$ & $\begin{array}{l}22.3 \\
(3.9)\end{array}$ & $\begin{array}{l}6.0 \\
(2)\end{array}$ & $\begin{array}{l}8.5 \\
(3.2)\end{array}$ \\
\hline $2(\mathrm{M})^{*}$ & 67 & 22 & $\mathrm{~L}$ & 6 & 15 & 1700 & 7 & 21.5 & 20.5 & 19 & 6 & 6.3 \\
\hline
\end{tabular}




\begin{tabular}{|c|c|c|c|c|c|c|c|c|c|c|c|c|}
\hline $5(\mathrm{M})^{*}$ & 67 & 11 & $\mathrm{R}$ & 5 & 5 & 150 & 20 & 34.5 & 31 & 23 & 7 & 6.4 \\
\hline $11(\mathrm{M})^{*}$ & 61 & 15 & $\mathrm{~L}$ & 5 & 7 & 810 & 21 & 43 & 36 & 13 & 7 & 5.3 \\
\hline $12(\mathrm{~F})^{*}$ & 72 & 12 & $\mathrm{~L}$ & 6 & 2 & 725 & 26 & 33 & 22 & 18 & 7 & 7.8 \\
\hline Mean & 66.8 & 15 & $3 \mathrm{~L} / 1 \mathrm{R}$ & 5.5 & 7.3 & 846 & 18.5 & 33 & 29.7 & 18.3 & 6.7 & 6.5 \\
$(\mathrm{SD})^{*}$ & $(4.5)$ & $(5)$ & & $(0.6)$ & $(5.6)$ & $(640)$ & $(8.1)$ & $(8.8)$ & $(7.1)$ & $(4.1)$ & $(0.5)$ & $(1)$ \\
\hline Grand & 64.3 & 14.2 & $5 \mathrm{R} / 7 \mathrm{~L}$ & 4.8 & 5.4 & 655 & 21 & 37 & 24.1 & 20.9 & 6.3 & 7.8 \\
Mean & $(4.1)$ & $(4.3)$ & & $(2.2)$ & $(3.5)$ & $(417)$ & $(7.4)$ & $(7.4)$ & $(5.9)$ & $(4.3)$ & $(1.7)$ & $(2.8)$ \\
$(\mathrm{SD})$ & & & & & & & & \\
\hline
\end{tabular}

*Patients who completed the study.

DBS = deep brain stimulation; FOG = freezing of gait; Grand Mean = mean of values referred to the whole study population (patients who completed and who not completed the study); LEDD = Levodopa equivalent daily dose; $\mathrm{L}=$ left; $\mathrm{R}=$ right; MDS UPDRS = Movement Disorder Society Unified Parkinson's disease Rating Scale; PDQ-SI = Parkinson's Disease Questionnaire-39 Summary Index Score; SD = Standard deviation. 
Table 2. Chronic (CHR) vs experimental (EXP) STN DBS settings of the study population.

\begin{tabular}{|c|c|c|c|c|c|c|c|c|c|}
\hline \multirow{2}{*}{ Patient, n. } & \multirow{2}{*}{ L contact } & \multirow{2}{*}{$\mathrm{R}$ contact } & \multicolumn{2}{|c|}{ L amplitude (V) } & \multicolumn{2}{|c|}{ R amplitude (V) } & \multirow{2}{*}{$\begin{array}{l}\text { L PW } \\
(\mu \mathrm{sec})\end{array}$} & \multirow{2}{*}{$\begin{array}{l}\text { R PW } \\
(\mu \mathrm{sec})\end{array}$} & \multirow{2}{*}{ Frequency $(\mathrm{Hz})$} \\
\hline & & & CHR & EXP & CHR & EXP & & & \\
\hline 1 & $5-$ & $1+2-$ & 2.2 & $1.55 \#$ & 2.8 & 2.8 & 60 & 60 & 130 \\
\hline 3 & $6-$ & $3-$ & 3.2 & 3.2 & 2.5 & $1.75 \#$ & 60 & 60 & 130 \\
\hline 4 & $6-$ & $2-$ & 3.4 & 3.4 & 2.4 & $1.65 \#$ & 60 & 60 & 130 \\
\hline 6 & $9-10+$ & $1-$ & 3.8 & $2.65 \#$ & 3.0 & 3.0 & 60 & 60 & 130 \\
\hline 7 & $2-$ & 9- & 2.2 & 2.2 & 3.2 & $2.25 \#$ & 60 & 60 & 130 \\
\hline 8 & $6-$ & $2-$ & 2.0 & $1.4 \#$ & 2.1 & 2.1 & 60 & 60 & 130 \\
\hline 9 & $10-$ & $2-$ & 3.6 & $2.50 \#$ & 3.0 & 3.0 & 60 & 60 & 80 \\
\hline 10 & $6-$ & $2-$ & 3.2 & 3.2 & 3.6 & $2.50 \#$ & 60 & 60 & 130 \\
\hline $2 *$ & $5-$ & $1-$ & 2.7 & 1.9\# & 2.7 & 2.7 & 60 & 60 & 130 \\
\hline $5^{*}$ & $6-$ & $2-$ & 3.0 & 3.0 & 3.2 & $2.2 \#$ & 60 & 60 & 130 \\
\hline $11 *$ & $5+6-$ & $2-$ & 4.4 & $3.1 \#$ & 2.5 & 2.5 & 60 & 60 & 130 \\
\hline $12 *$ & $6-$ & $2-$ & 3.4 & 2.4\# & 3.2 & 3.2 & 60 & 60 & 180 \\
\hline $\begin{array}{l}\text { Mean } \\
(\mathrm{SD})\end{array}$ & & & $3.0(0.70)$ & & $2.9(0.4)$ & & $60(0)$ & $60(0)$ & $130(21.3)$ \\
\hline
\end{tabular}


* Patients who completed the study.

\# STN with reduced Voltage.

$\mathrm{CHR}=$ chronic $; \mathrm{EXP}=$ experimental $\mathrm{Hz}=$ Hertz $; \mathrm{L}=$ left $; \mathrm{R}=$ right $; \mathrm{PW}=$ pulse width $; \mathrm{SD}=$ standard deviation; $\mathrm{V}=$ voltage; 
Table 3. Secondary outcomes: individual scores in EXP and CHR conditions in patients who completed the study.

\begin{tabular}{|c|c|c|c|c|c|c|c|c|c|c|}
\hline & $\begin{array}{r}\text { MDS-U } \\
\text { MED }\end{array}$ & $\begin{array}{l}\text { DRS III } \\
\text { OFF }\end{array}$ & $\begin{array}{r}\text { MDS-U } \\
\text { MEI }\end{array}$ & $\begin{array}{l}\text { RS III } \\
\text { ON }\end{array}$ & MDS-UI & DRS II & FOG- & score & PDQ- & score \\
\hline Patient, $\mathrm{n}$. & CHR & EXP & CHR & EXP & CHR & EXP & CHR & EXP & CHR & EXP \\
\hline 2 & 27 & 27 & 32 & 37 & 5 & 9 & 19 & 19 & 7.1 & 7.1 \\
\hline 5 & 28 & 36 & 21 & 27 & 10 & 14 & 15 & 20 & 5.9 & 4.6 \\
\hline 11 & 42 & 38 & 36 & 29 & 19 & 23 & 16 & 19 & 5.9 & 3.4 \\
\hline 12 & 34 & 33 & 22 & 26 & 20 & 12 & 13 & 11 & 7.3 & 7.1 \\
\hline $\begin{array}{l}\text { Mean } \\
\text { (SD) }\end{array}$ & $32.8(6.9)$ & $33.5(4.8)$ & $27.8(7.4)$ & $29.8(5)$ & $13.5(7.2)$ & $14.5(6.0)$ & $16.8(2.5)$ & $19.3(4.2)$ & $6.5(0.8)$ & $5.6(1.9)$ \\
\hline
\end{tabular}

FOG-Q = freezing of gait questionnaire; MDS UPDRS = Movement Disorder Society Unified Parkinson's disease Rating Scale; PDQ-SI =

Parkinson's Disease Questionnaire-39 Summary Index Score. 


\section{Figure Legend}

Figure 1. Percentage of FOG in the chronic (CHR) and experimental (EXP) stimulation conditions, in the OFF medication condition (A), in the

ON medication condition (B), and composite gait score under CHR and EXP conditions (C).

In color on the Web and in black-and-white in print. 
Figure 1.
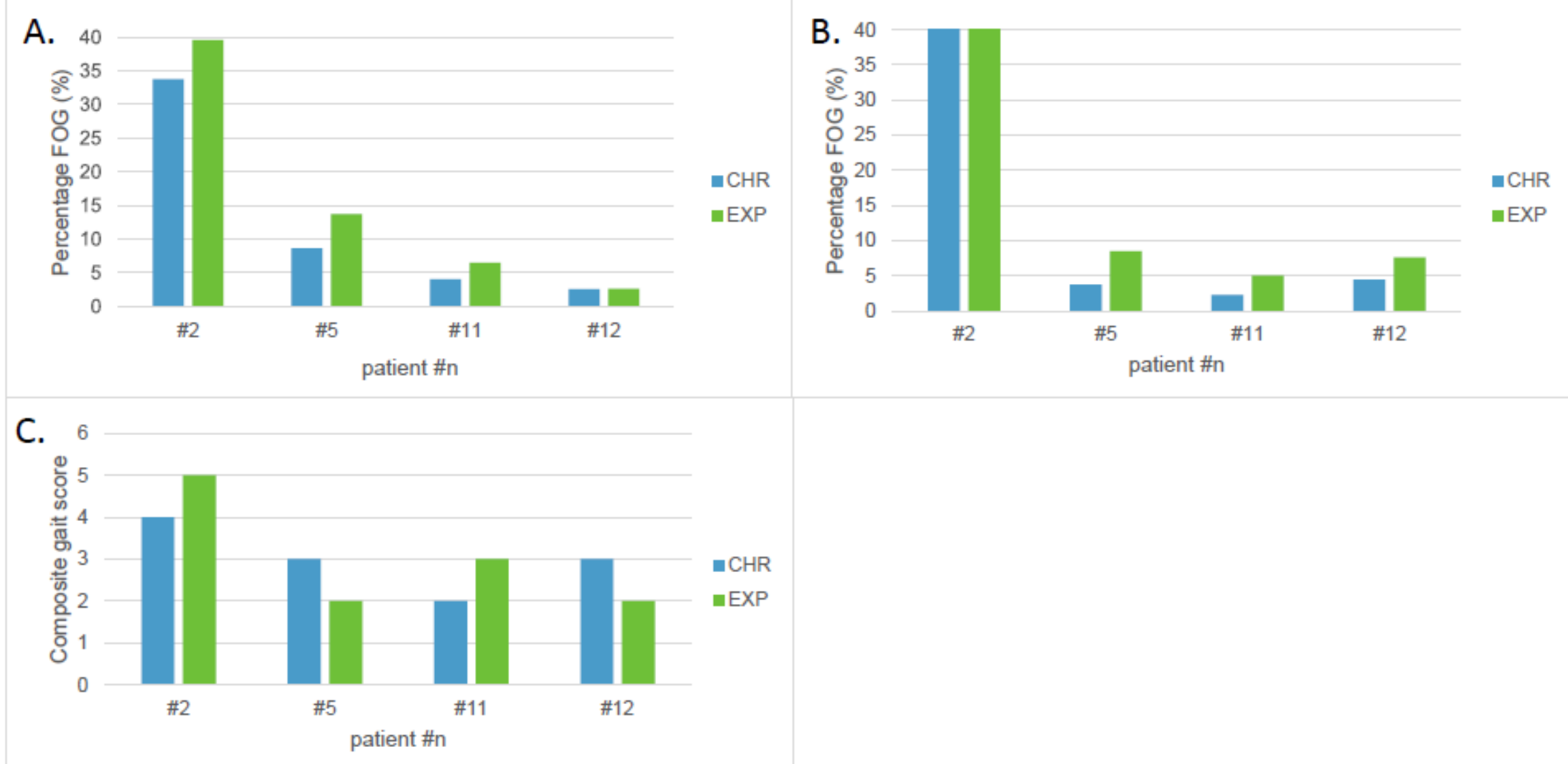